\title{
Lower hybrid waves at the shock front: a reassessment
}

\author{
S. N. Walker ${ }^{1}$, M. A. Balikhin ${ }^{1}$, H. St. C. K. Alleyne $^{1}$, Y. Hobara ${ }^{1}$, M. André ${ }^{2}$, and M. W. Dunlop ${ }^{3}$ \\ ${ }^{1}$ Department of Automatic Control and systems Engineering, University of Sheffield, Sheffield, UK \\ ${ }^{2}$ Swedish Institute for Radio Physics-Uppsala Division, University of Uppsala, Sweden \\ ${ }^{3}$ Space Physics Division, SSTD, CCLRC Rutherford Appleton Laboratory, Didcot, Oxfordshire, UK
}

Received: 5 September 2007 - Revised: 17 January 2008 - Accepted: 25 February 2008 - Published: 26 March 2008

\begin{abstract}
The primary process occurring at a collisionless shock is the redistribution of the bulk upstream energy into other degrees of freedom. One part of this process results in the acceleration of electrons at the shock front. Accelerated electrons are observed at the terrestrial and other planetary shocks, comets, and their effects are observed in astrophysical phenomena such as supernova remnants and jets in the form of X-ray bremsstrahlung radiation. One of the physical models for electron acceleration at supercritical shocks is based on low-hybrid turbulence due to the presence of reflected ions in the foot region. Since lower hybrid waves propagate almost perpendicular to the magnetic field they can be simultaneously in resonance with both the unmagnetised ions $\left(\omega=V_{i} k_{\perp}\right)$ and magnetised electrons $\left(\omega=V_{e} k_{\|}\right)$. In this paper, Cluster observations of the electric field are used to study the occurrence of lower hybrid waves in the front of the terrestrial bow shock. It is shown that the lower hybrid waves exist as isolated wave packets. However, the very low level of the observed lower hybrid turbulence is too small to impart significant energisation to the electron population.
\end{abstract}

Keywords. Space plasma physics (Shock waves; Waveparticle interactions; Waves and instabilities)

\section{Introduction}

The energisation of electrons within a collisionless shock requires the transfer of a portion of the energy associated with the incoming upstream plasma flow to the electron population. In order for this energy transfer to occur, there has to be some media that can channel energy from the incoming ion population to the electrons. One mechanism that has commonly been proposed, both for solar systems and particularly for astrophysical applications is based on excitation of lowerhybrid waves (Laming, 2001).

Correspondence to: S. N. Walker

(simon.walker@sheffield.ac.uk)
The lower-hybrid wave is an electrostatic plasma wave mode whose plasma frame frequency is in the vicinity of the lower-hybrid resonance frequency $\omega_{l h} \sim \sqrt{\omega_{c i} \omega_{c e}}$ where $\omega_{c i}$ and $\omega_{c e}$ are the proton and electron gyrofrequencies, respectively. The wave has linear polarisation and propagates almost perpendicular with respect to the magnetic field $\left(\cos \left(\theta_{k B}\right) \sim \sqrt{m_{e} / m_{i}} \sim 89^{\circ}\right)$. The maximum growth rate $\gamma_{\text {MAX }}$ occurs when $k_{\| \mid} / k \sim \omega_{p i} / \omega_{p e}$.

Since the waves are propagating in a plasma that is moving with respect to the satellite, their frequencies will be Doppler shifted in the spacecraft frame. The magnitude of this shift can be estimated using the resonance condition of the Modified Two Stream Instability (MTSI) $2 V_{A} M_{A} k=\omega_{l h}$. This gives a maximum estimate for the correction in observed wave frequency due to the Doppler shift $k V_{s w} \sim \omega_{l h} / 2$.

Current models base the occurrence of lower hybrid waves at a shock front on counter-streaming populations of ions which result from either the reflection of ions (Leroy et al., 1982) at the front via the MTSI or mass-loading process. These models are often used to explain the electron acceleration observed at various astrophysical shocks such as supernova remnants (Laming, 2001). However, there is currently no substantial experimental evidence that these waves do indeed exist in the fronts of supercritical, quasiperpendicular, collisionless shocks. The results of data analysis from the Intershock electric field experiment, in which wave activity was observed at frequencies of a few Hertz, has been used to argue for the existence of lower hybrid waves. An alternative explanation, however, has been proposed in which Intershock may have simply observed the electric field component of whistler wave packets propagating in the foot region (Balikhin et al., 1997; Walker et al., 1999). Electric field observations of Comet Halley also showed evidence for waves observed in the vicinity of the lower hybrid frequency (Klimov et al., 1986). However, their exact wave mode was not determined. 


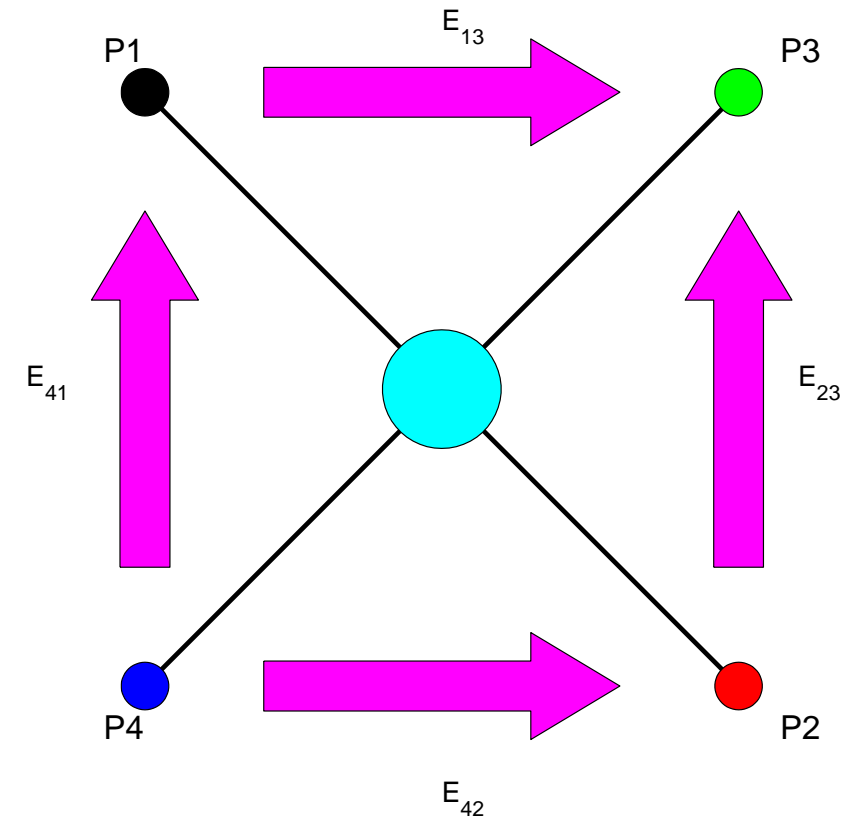

Fig. 1. The configuration of the EFW electric field probes.

Wave activity in the electric field at frequencies of a few hertz is usually attributed to one of two modes, either the whistler (Balikhin et al., 1997) or lower hybrid (Vaisberg et al., 1983). One method to differentiate these modes is to examine their polarisations. Whistler mode waves are elliptically polarised whilst lower hybrid waves, as mentioned above, are linearly polarised. The remainder of this paper reports Cluster observations of waves around the lower hybrid frequency at the terrestrial bow shock to accesses the level of lower hybrid turbulence in the foot and its possible effect on the electron acceleration. Section 2 describes the data source and methodology used for this study. Results are presented in Sect. 3 and discussed in relation to the acceleration of electrons in Sect. 4. Finally, a summary of the main findings is presented in Sect. 5.

\section{Data source and methodology}

The primary data set used in this study was collected by the EFW instrument (Gustafsson et al., 1997) onboard the Cluster satellites using onboard timing provided by the DWP instrument (Woolliscroft et al., 1997). EFW uses two pairs of spherical probes in the satellite spin plane situated on the ends of wire booms whose length is $44 \mathrm{~m}$ as shown in Fig. 1 . Thus, the distance between probes adjacent/opposite to one another is $\sim 62 / 88 \mathrm{~m}$ respectfully. Normally, the EFW instruments return the electric field calculated as the difference in probe potentials between probes 1 and $2\left(E_{12}\right)$ and 3 and $4\left(E_{34}\right)$ with a sampling rate of either $25 \mathrm{~Hz}$ (normal science mode) or $450 \mathrm{~Hz}$ (burst science mode). The individual probe potentials are also available with a time resolution of $5 \mathrm{~Hz}$. In addition to these standard modes, there is a triggered internal burst mode. Using this mode, data for a short time period may be captured with a much higher sampling rate. The EFW data that has been analysed in this study consists of internal burst mode data comprising the four individual probe potentials sampled at $9 \mathrm{kHz}$ for periods of around $10 \mathrm{~s}$. Since the internal burst data is captured and stored depending upon some criteria, it may be that although the shock region was targeted for data collection, the waveforms returned may not have been captured in the shock front itself. To this end, a search was made to find possible candidate events by cross referencing the list of Cluster shock crossings for 2002 with the list of periods for which internal burst data are available. This resulted in a list of 10 possible events. Of these events, a comparison between the FGM magnetic field measurements and the time periods for which EFW internal burst data were available showed that there were only two shocks for which the period of internal burst data lay solely in the foot region of the shock. Of these, one shock possessed a magnetic profile that was highly turbulent and difficult to interpret and was also eliminated from further analysis. This left just one clean shock on which to perform the analysis.

As mentioned above, the internal burst data sets are the only ones generated by EFW that contain the individual probe potentials at a high enough sampling rate to investigate waves and turbulence at frequencies around the lowerhybrid frequency in the vicinity of the terrestrial bow shock $(10-30 \mathrm{~Hz})$. By using the individual probe potentials it is possible to compute two parallel electric field components one on either side of the satellite. For example, the probe pairs 1,3 and 4,2 maybe used to compute electric field components $\mathrm{E}_{13}$ and $\mathrm{E}_{42}$ whose directions are parallel and are spatially separated by a distance of $\sim 62.2 \mathrm{~m}$. This technique has previously been used by Balikhin et al. (2005) and Tjulin et al. (2003) to study small scale electric field structures and waves and is similar to the short baseline interferometry techniques employed in the analysis of data from sounding rockets (Pinçon et al., 1997).

Since the probe potentials can be used to calculate two parallel electric field vectors it should be possible to use the phase differencing technique (Balikhin and Gedalin, 1993) in order to compute the component of the wave vector $k$ that lies in the satellite spin plane perpendicular to the electric field components measured on either side of a single satellite. This technique assumes that the wave field within the plasma $B(r, t)$ may be decomposed as the sum of individual waves:

$B(\boldsymbol{r}, t)=\Sigma_{\text {mode }} \Sigma_{\omega} \exp (i \boldsymbol{k} \boldsymbol{r}-i \omega t)+C C$

Thus, the phase difference measured at two closely spaced points is related to the projection of the wave vector $k$ along the separation vector between the two measurement points.

$\Delta \Phi(\omega)=\Psi_{1}-\Psi_{2}=\boldsymbol{k}(\omega) \boldsymbol{r}_{1}-\boldsymbol{k}(\omega) \boldsymbol{r}_{2}=\boldsymbol{k}(\omega) \Delta \boldsymbol{r}$ 
The resulting histogram of phase differences as a function of $k$ and wave frequency $\omega$, known as the joint $\omega-k$ spectrogram, shows the dispersion relation of the waves in the spacecraft frame. By combining the dispersions for two mutually perpendicular sets of electric field components it is possible to determine the component of the wave vector $k$ that lies in the satellite spin plane.

The phase differencing method may also be used to examine the polarisation characteristics of the wave in question. In this case, the phase differencing algorithm is applied to a pair of perpendicular components of the electric field (as opposed to the parallel field components mentioned above). The resulting histogram of the phase difference as a function of frequency yields a vertical line of constant phase difference with respect to frequency at a phase difference of zero for a linearly polarised wave and $\pm \pi / 2$ for a circularly polarised wave. Thus, this technique may be used to help distinguish between a linearly polarised lower hybrid wave and a circularly polarised whistler mode wave, both of which have been observed at these frequencies. This method is used in preference to an examination of the coherency (see for example Krasnosel'skikh et al., 1991) due to the short duration of the wave packets.

The magnetometer data, used to put the electric field measurements into context within the shock front and compute the lower hybrid resonance frequency, come from the FGM instruments (Balogh et al., 1997) and made publically available through the Cluster Active Archive. These measurements typically have a sampling rate of $22 \mathrm{~Hz}$.

\section{Results}

Figure 2 shows an overview of the magnetic profile of the shock encountered on 26 February at 21:34 UT. From Fig. 2 it can be seen that Cluster 3 first encountered the foot region of the shock just before 21:34 UT, finally crossing the ramp and entering the downstream region at approximately 21:34:15 UT. For this shock encounter, the EFW internal burst data selection was triggered at 21:34:01.922 UT and lasted for a period of $10.47 \mathrm{~s}$ as indicated by the shaded region in the figure.

As mentioned above, the analysis presented in this paper was performed on data recorded on 26 February 2002 just after 21:34 UT on spacecraft 3 . This quasi-perpendicular shock crossing took place on an inbound pass at a position (12.0, $-1.60,8.07) R_{e}$. As can be seen from Fig. 2 the whole period of internal burst data was collected in the foot region of the shock. The initial increase in the magnetic field profile at around 21:34 UT has been shown by Balikhin et al. (2005) to be part of the foot region rather than a partial ramp crossing.

During the 10.5-s period for which EFW internal burst data is available there were several short periods when the electric field measurements indicated that there were oscillations occurring at or just above the local lower-hybrid reso-

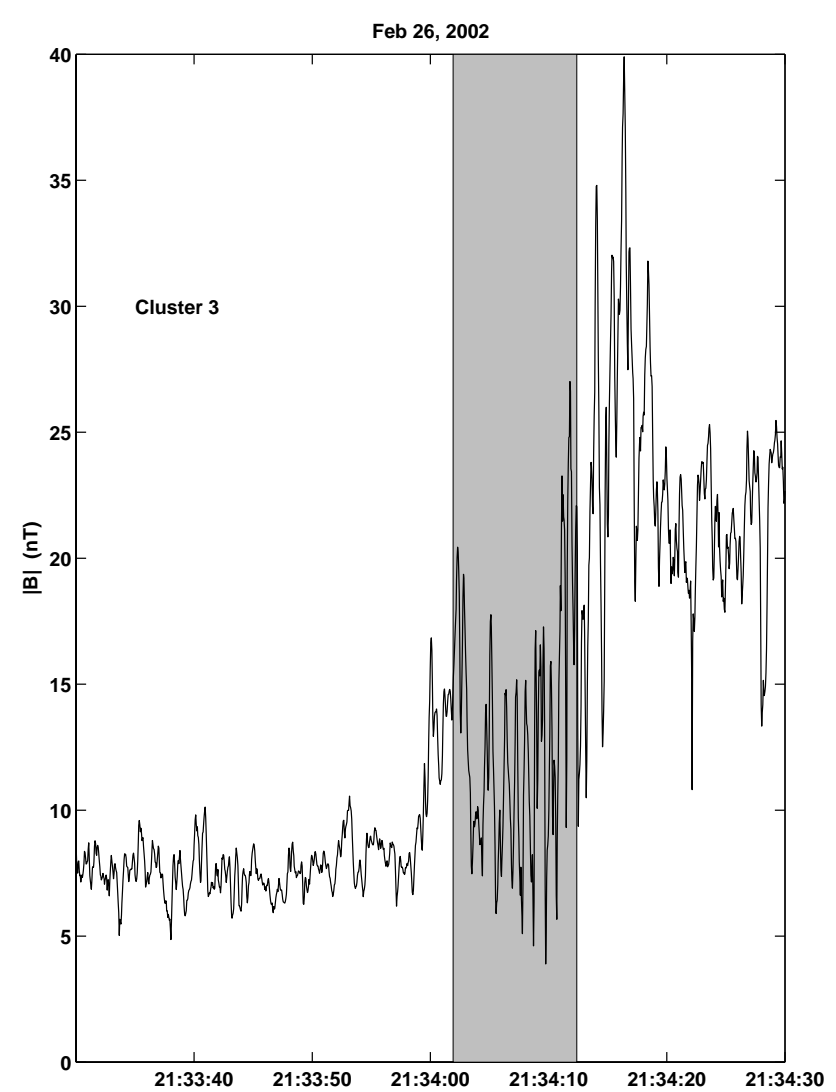

Fig. 2. The magnetic profile of the bow shock crossing observed by Cluster 3 on 26 February 2002 just after 2134UT. The period for which EFW internal burst mode data is available is indicated by the shaded region.

nance frequency. In the following subsections the properties of the waves in three such events are discussed.

\subsection{Event 1}

The first event presented in this paper occurred just after 21:34:05 UT. Figure 3 shows a dynamic spectrogram of the electric fields $E_{12}$ (top) and $E_{34}$ measured between probes $P_{1}$ and $P_{2}$ and probes $P_{3}$ and $P_{4}$, respectively, calculated using a Morlet wavelet transform. The black line represents the lower-hybrid resonance frequency. It is clearly seen that at around 21:34:05.2 and there is a packet of waves at a frequency between $10-20 \mathrm{~Hz}$, whose lower edge is just above the lower-hybrid resonance frequency. The duration of this wave packet is around $3 \mathrm{~ms}$ which corresponds to a few wave periods. Having identified a possible occurrence of lower hybrid waves, the phase differencing technique was applied to parallel electric field vectors in an attempt to compute the dispersion relation of the waves and hence provide an unambiguous identification of the wave mode. 


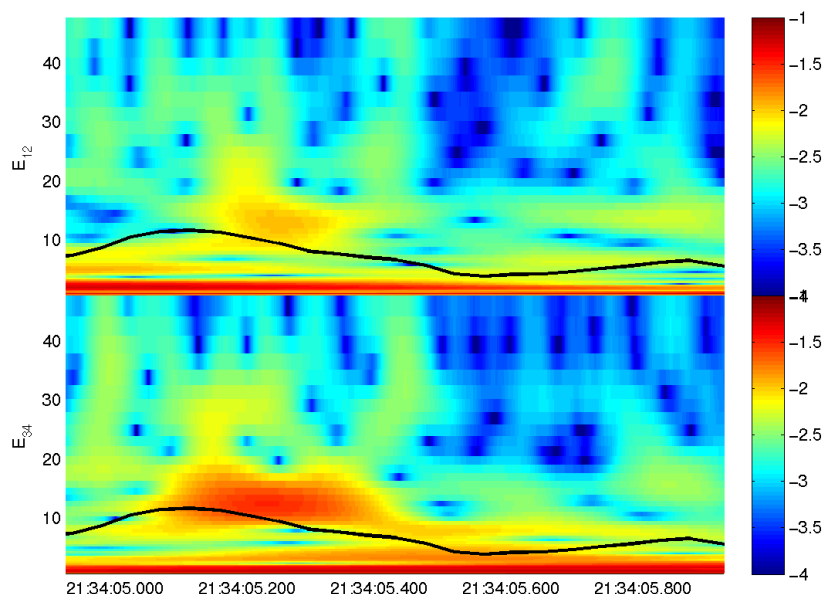

Fig. 3. The wavelet dynamic spectrogram of electric fields $E_{12}$ (top) and $E_{34}$ showing a the occurrence of oscillations just above the lower-hybrid resonance frequency (black line) for event 1 .

The joint $\omega-k$ spectrum generated using the phase differencing method is shown in Fig. 4 using the spin plane electric field components $E_{13}$ and $E_{42}$. These electric field measurements represent two parallel component directions (as indicated in Fig. 1). Thus, the resulting dispersion represents wave field $k$-vector component in the direction perpendicular to these electric field directions. As can be seen from Fig. 4 in the frequency range of interest $(10-20 \mathrm{~Hz})$ no measurable dispersion of the waves is observed on scales of the separation distance of the probe pairs $(62.2 \mathrm{~m})$. This implies that the wave travels over the spacecraft at rather high speed so that there is virtually no difference in the phase of the wave measured at the two points on either side of the satellite. This is also evident in the waveform of the electric field signals shown in Fig. 5. The two waveforms in each panel represent parallel components of the electric field, ie $E_{13}$ and $E_{42}$ in the top panel, $E_{14}$ and $E_{32}$ in the lower panel. A comparison of the two curves within a panel shows that the two measurements which are observed to vary in phase which would indicate that whatever passed over the satellite has a scale much larger than the individual probe separation distances.

Since the phase differencing method is appears unable to show any dispersion in the parallel electric field vectors this method cannot be used to reliably identify the wave packet as being lower hybrid. As a result, we need to investigate some other wave properties of the wave packet to determine if they are compatible with the lower hybrid mode. It was mentioned in Sect. 2 that by applying the phase differencing method to perpendicular components of the electric field it should be possible to determine whether the wave packet is linearly or circularly polarised. The $\omega-k$ spectrum calculated using the electric field vectors $E_{13}$ and $E_{41}$ is shown in Fig. 6. In the frequency range $10-20 \mathrm{~Hz}$ the value of the phase difference is around zero. This result indicates that the

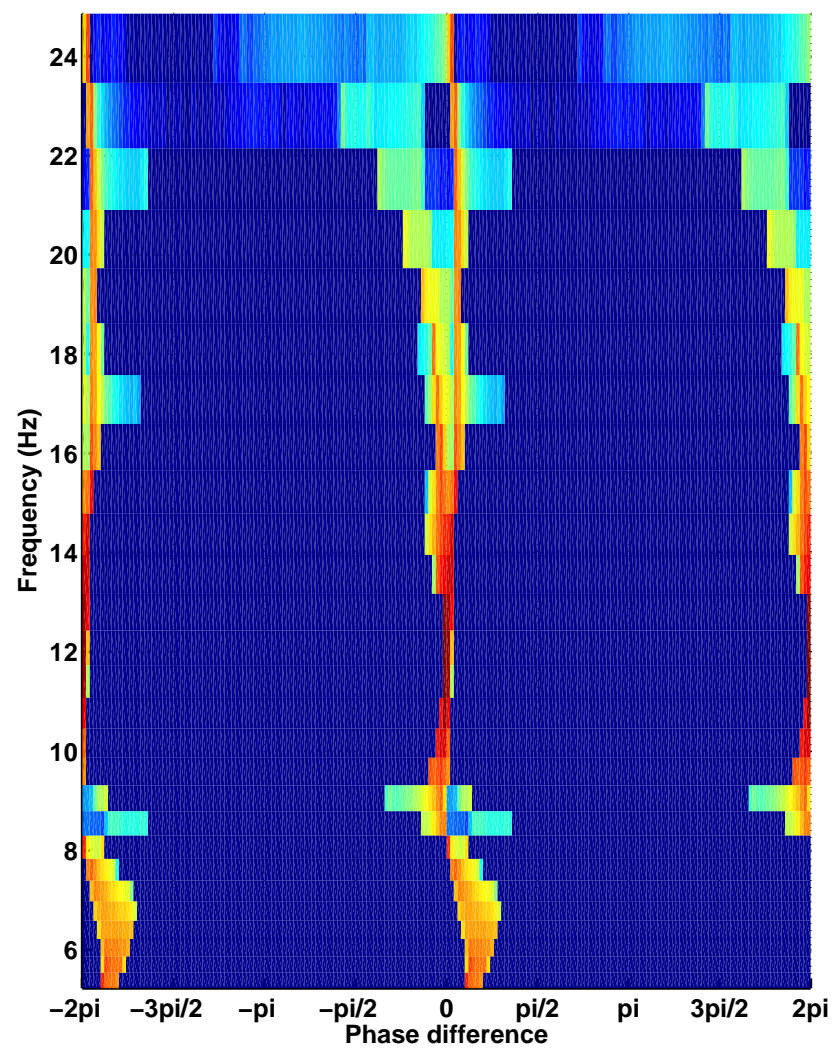

Fig. 4. The $\omega-k$ joint spectrum calculated from the phase differences measured between the two parallel electric field components $E_{13}$ and $E_{42}$ for event 1.

wave possesses linear polarisation, a result consistent with that of a lower-hybrid mode wave. This result can also be seen in the waveform plots (Fig. 5 where it can be observed that the variations observed in $E_{12}$ and $E_{42}$ (top panel) are mirrored by those in the lower panel. Thus we can conclude that the wave mode that we are observing is consistent with a lower-hybrid mode.

\subsection{Event 2}

The second event to be discussed here occurred around 21:34:04.5 on 26 February 2002. The wavelet dynamic spectra for this period, displayed in Fig. 7, shows a wave packet occurring on the frequency range $10-20 \mathrm{~Hz}$. The black line, which represents the lower hybrid resonance frequency, lies on the lower frequency border of this packet which is typical of frequency range expected for lower hybrid mode waves. Figure 8 shows the joint $\omega-k$ wave spectrum computed with the electric field signals $E_{13}$ and $E_{42}$ for this wave packet. In the frequency range of interest $(10-20 \mathrm{~Hz})$ there is a clear ridge at a phase difference of zero which again indicates that the wave is propagating with a large phase speed over the satellite. This result is also evident from the electric field 


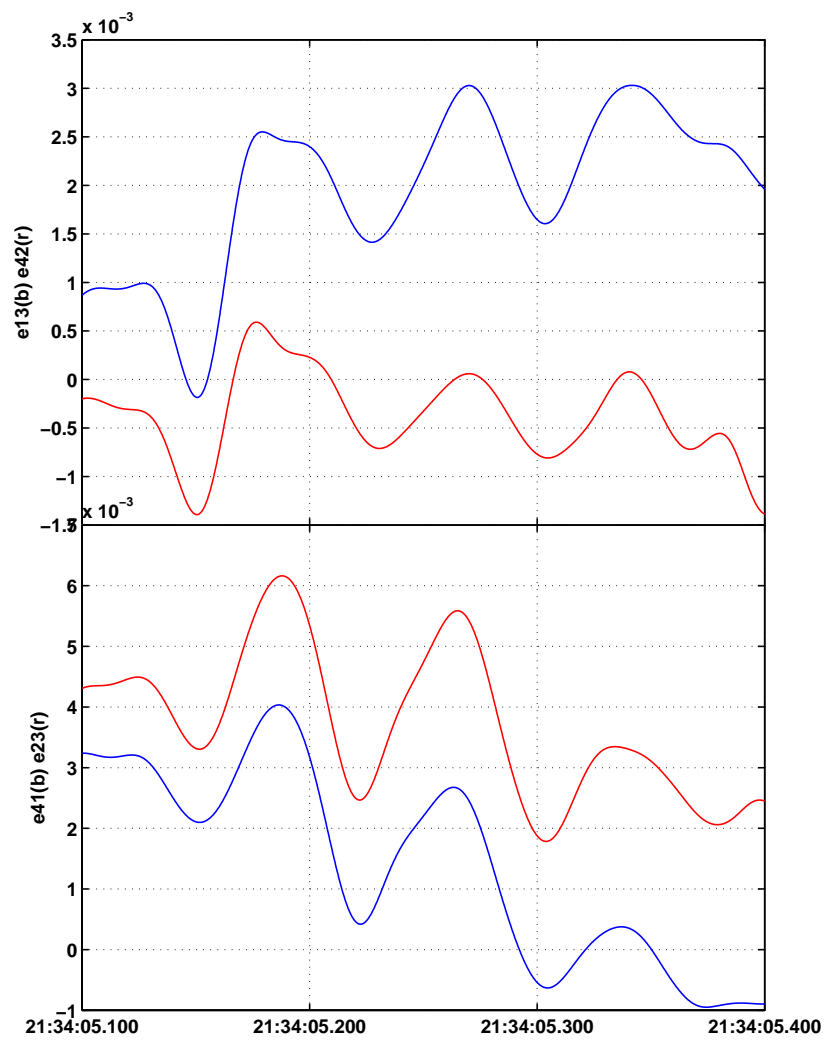

Fig. 5. The electric field waveforms $E_{13}$ and $E_{42}$ (top panel) and $E_{41}$ and $E_{23}$ (lower panel) for the first event.

waveforms for this packet that are plotted in Fig. 9. The polarisation of this wave packet was also investigated using the phase differencing method applied to two perpendicular components of the electric field. The result, shown in Fig. 10, shows a ridge-like maximum at a phase difference of zero in the frequency range of interest thus indicating that the packet possesses a linear polarisation. This result is also evident in the electric field waveforms shown in Fig. 9. Once again, it appears that this wave packet exhibits properties that are consistent with propagation in the lower hybrid mode.

\subsection{Event 3}

The final event highlighted in this paper occurred between 21:34:07.3 and 21:34:07.45 UT on 26 February 2002. The wavelet dynamic spectrogram (Fig. 11) shows a wave packet in the frequency range $10-15 \mathrm{~Hz}$ that lies just above the lower hybrid resonance frequency (indicated by the black line). This wave packet is observed to drift in frequency as time increases. This change in frequency mirrors the change in the lower hybrid resonance frequency as calculated from the magnetic field. Analysis of parallel electric field components using the phase differencing method (Fig. 12) again indicates zero phase difference between these compo-

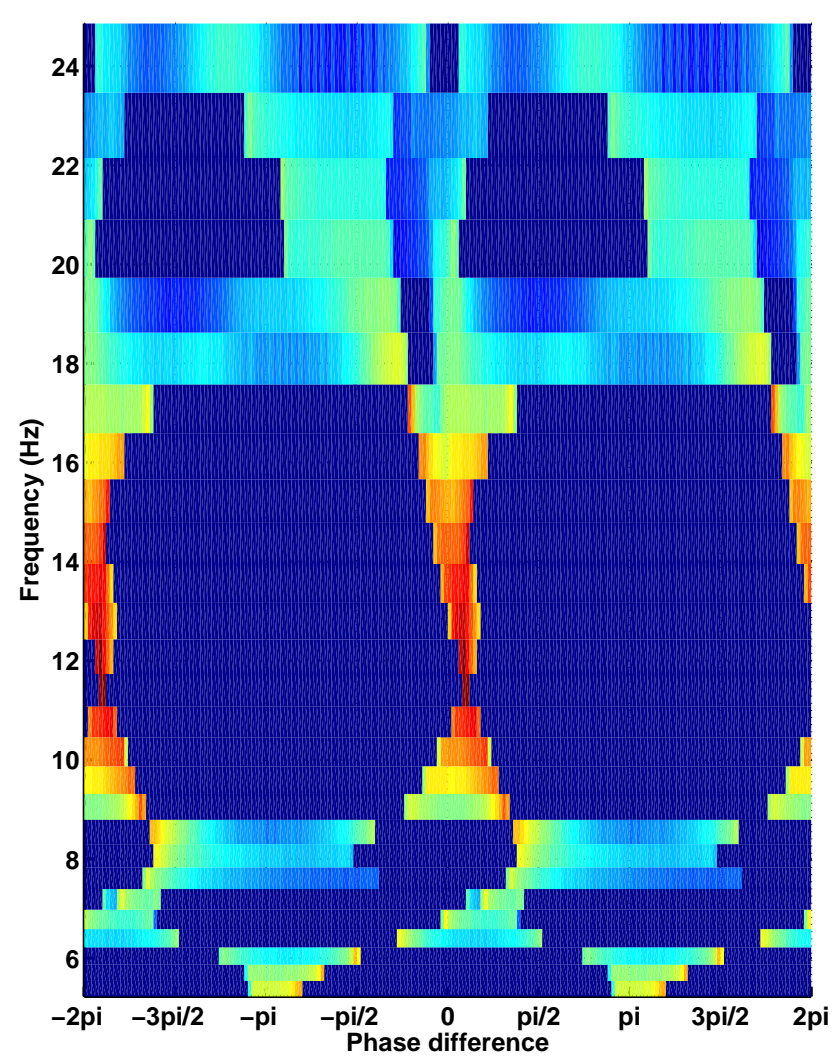

Fig. 6. The $\omega-k$ joint spectrum calculated from the phase differences measured between the two perpendicular electric field components $E_{13}$ and $E_{41}$ for the first event.

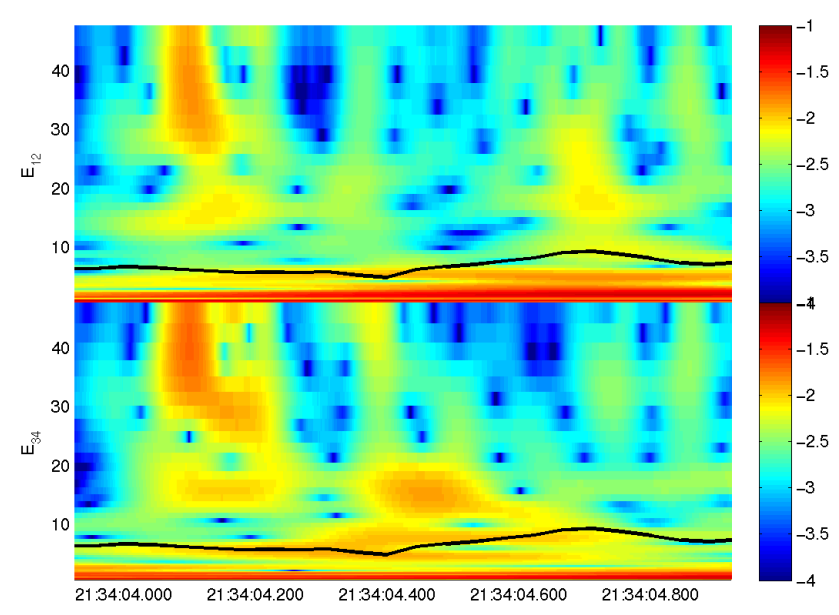

Fig. 7. The wavelet dynamic spectrum for event 2 (using the same format as Fig. 3

nents, a result evident from their waveforms that are shown in Fig. 13. However, a close comparison of perpendicular electric field components (i.e. comparison of the waveforms shown in the top panel with those in the lower panel) 


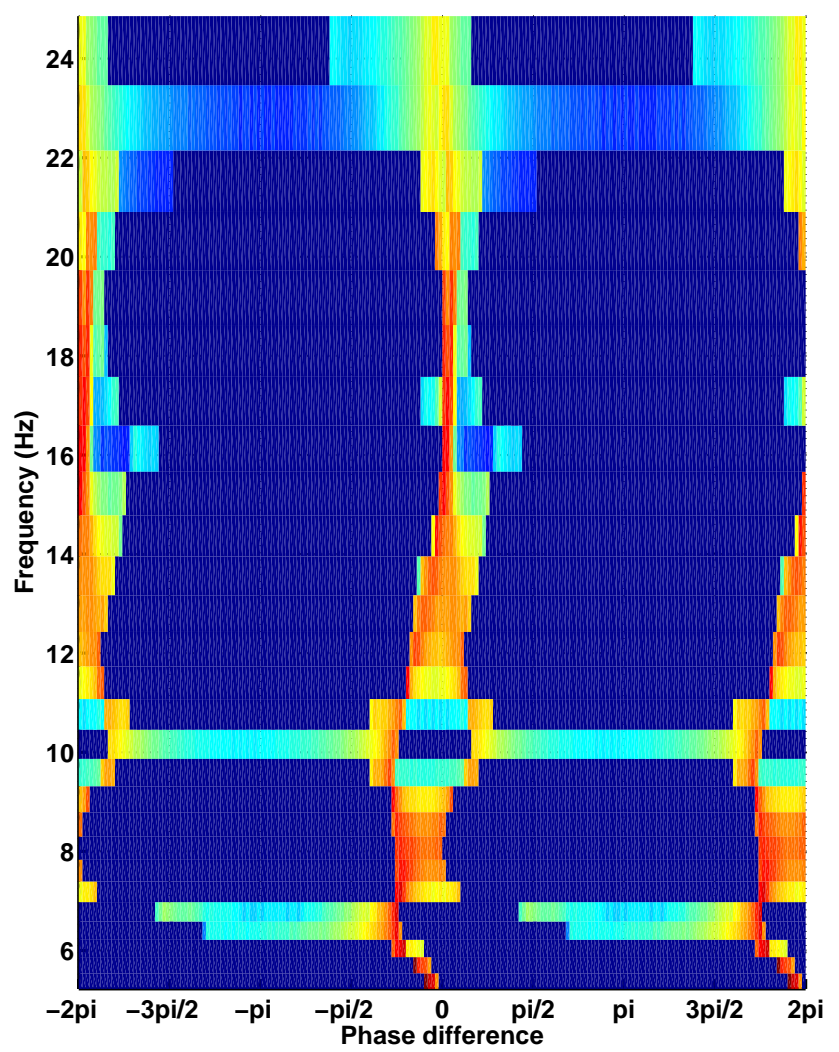

Fig. 8. The $\omega-k$ joint spectrum calculated from the two parallel electric field components $E_{41}$ and $E_{23}$ for event 2.

indicates a phase difference between the signals. This phase difference was further investigated using the phase differencing method. The joint $\omega-k$ spectrum for two perpendicular electric field components is shown in Fig. 14. In contrast to those for events 1 and 2 , event 3 produces a ridgelike maximum at a phase difference of $\pi / 2$. This confirms the conclusion reached by comparing the waveforms. This wave packet possesses circular polarisation and is thus not propagating in the lower hybrid mode. The circular polarisation indicates that this particular wave packet is propagating in the whistler mode.

Using a similar scenario, Krasnoselskikh et al. (1985) analysed the generation of fast electrons beams at quasiperpendicular shocks to explain the properties of Langmuir waves in the foreshock region. These authors conclude that in order to explain the measured brightness temperature of the Langmuir waves, the size of the electron acceleration region should be of the order of the radius of curvature of the shock front $\left(\sim 10^{6}\right) \mathrm{km}$. Again, this estimate for the size of the acceleration region is vastly grater than the spatial scale on which the lower hybrid wave packets are observed.

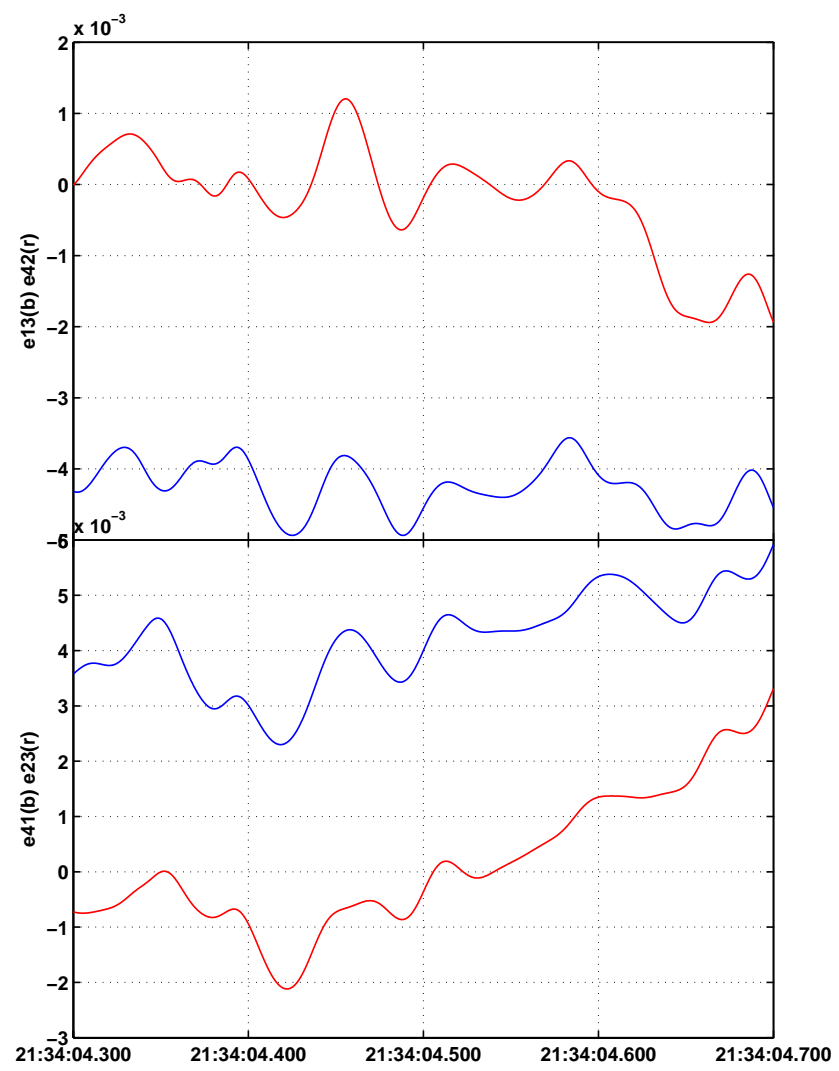

Fig. 9. The electric field waveforms $E_{13}$ and $E_{42}$ (top panel) and $E_{41}$ and $E_{23}$ (lower panel) for the second event.

\section{Discussion}

The observational evidence for the occurrence of lower hybrid waves in the foot region has been presented in the previous section. Waves with characteristics consistent with the lower hybrid mode were observed however, from the results shown in the previous section not all wave packets that occur just above the lower hybrid resonance frequency are propagating in the lower hybrid wave mode. Some of the wave packets were circularly polarised and are therefore whistler mode waves.

In the introduction it was mentioned that the occurrence of these waves form a crucial step in the mechanism for accelerating a small portion of the electron population to high energies. In this section, the observations are examined to determine if the lower hybrid waves can account for the occurrence of the high energy electrons.

At a collisionless shock, lower hybrid mode waves are generated by the MTSI, created when the incoming population of particles interacts with the small $(\sim 20 \%)$ of particles that have been reflected at the shock. Based on the occurrence of a beam-like ion population to provide the free energy for the low frequency plasma oscillations, the dispersion 


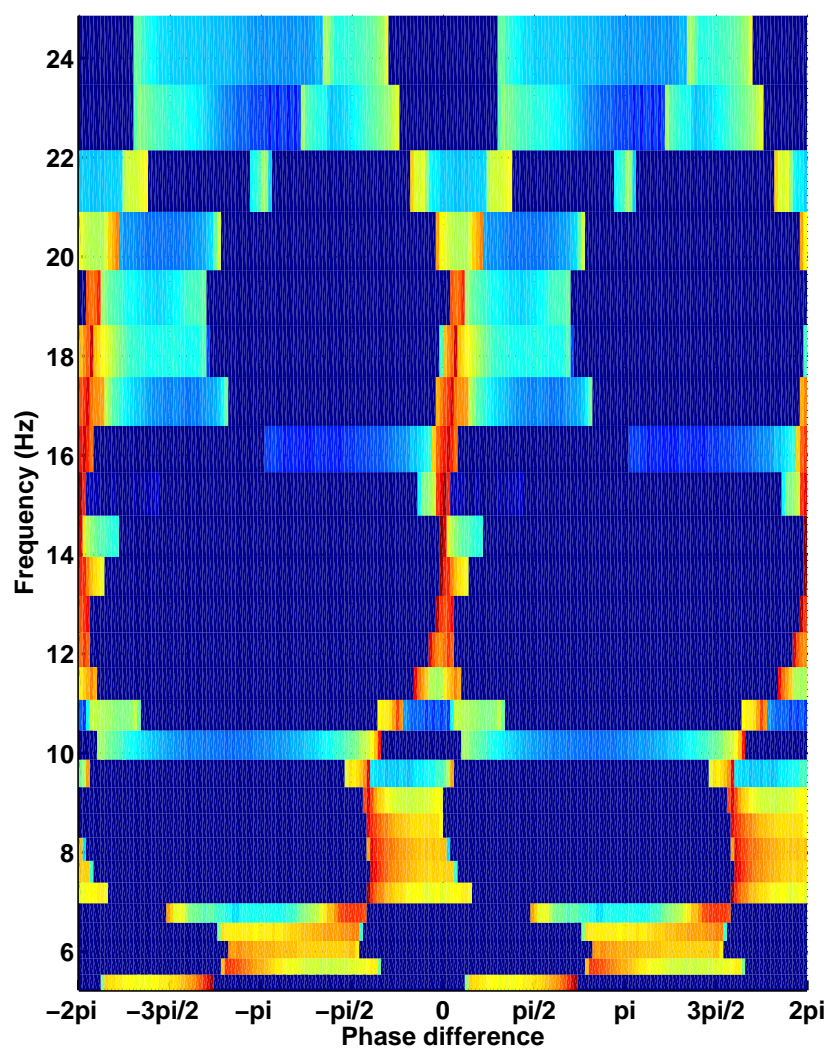

Fig. 10. The $\omega-k$ joint spectrum calculated from the two perpendicular electric field components $E_{42}$ and $E_{23}$ for the second event.

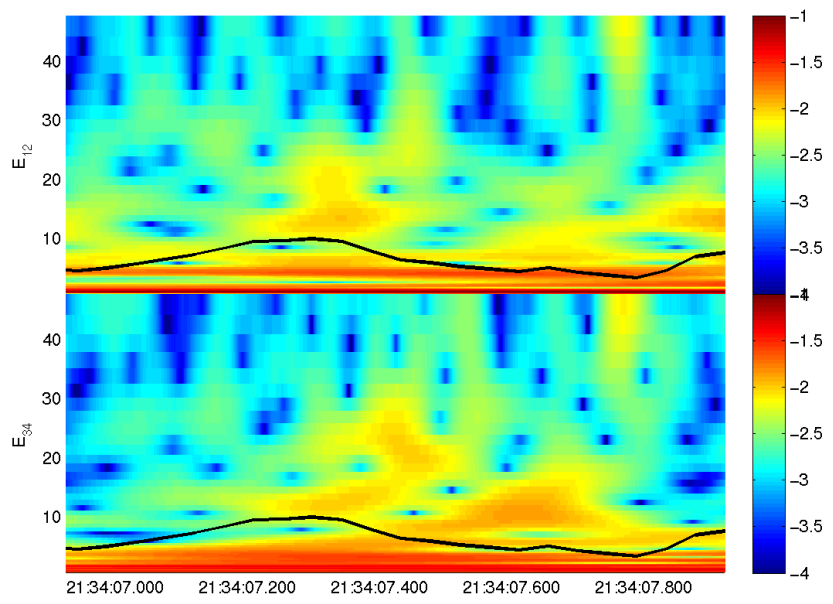

Fig. 11. The wavelet dynamic spectrum for event 3 (using the same format as Fig. 3.

relation for low frequency waves propagating almost perpendicular to the external magnetic field may be written in the

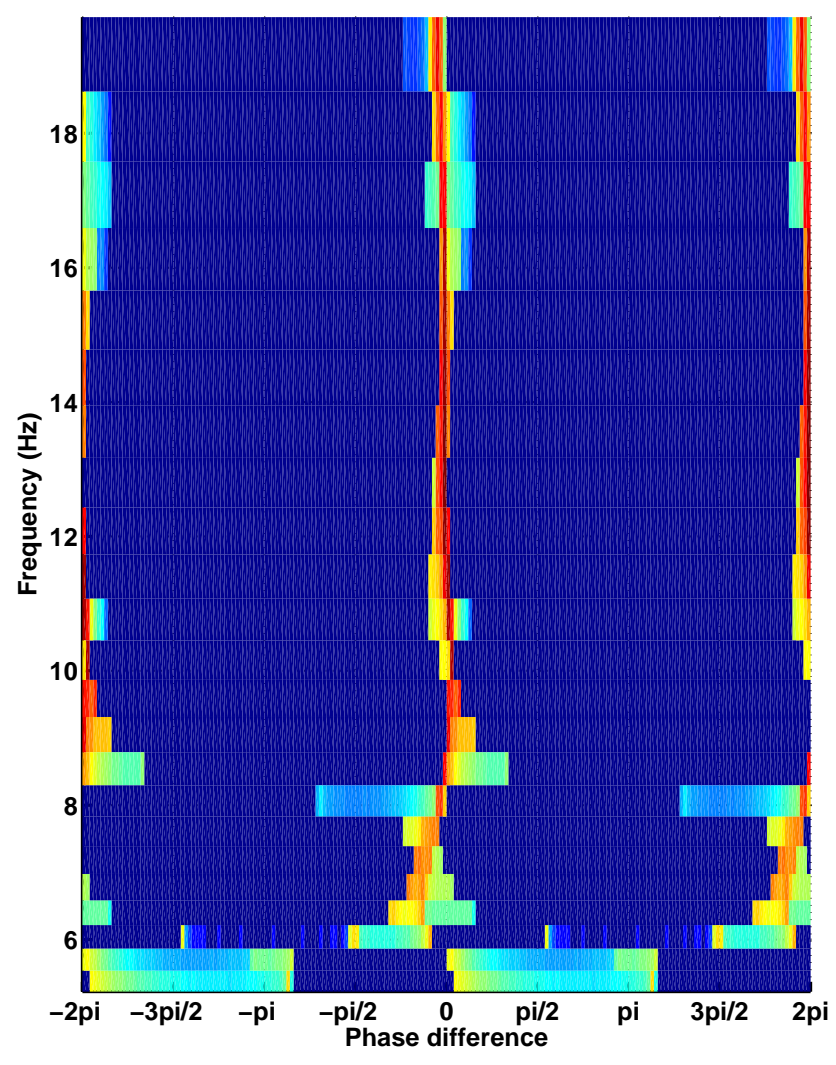

Fig. 12. The $\omega-k$ joint spectrum calculated the two parallel electric field components $E_{13}$ and $E_{42}$ for event 3 .

form (Vaisberg et al., 1983)

$$
\begin{aligned}
0 & =1+\frac{\omega_{p e}^{2} k_{\perp}^{2}}{\omega_{c e}^{2} k^{2}}\left(1+\frac{\omega_{p e}^{2}}{k_{\perp}^{2} c^{2}}\right)-\frac{\omega_{p i}^{2}}{\omega^{2}} \\
& -\frac{\omega_{p e}^{2} k_{\|}^{2}}{\omega^{2} k_{\perp}^{2}}\left(1+\frac{\omega_{p e}^{2}}{k_{\perp}^{2} c^{2}}\right)^{-1} \\
& +\frac{\omega_{p i}^{2}}{k^{2} n_{0}} \int \frac{\boldsymbol{k} \partial f_{b} / \partial \boldsymbol{v}}{\omega-\boldsymbol{k} \boldsymbol{v}+0 i} d^{3} \boldsymbol{v} \\
& +\frac{\omega_{p e}^{2}}{k^{2} n_{0}} \int \frac{k_{\|} \partial f_{T e} / \partial v_{\|}}{\omega-k_{\|} v_{\|}+0 i} d^{3} v_{\|}
\end{aligned}
$$

where $\omega_{p j}$ is the plasma frequency of species $j, \omega$ is the wave frequency, $\boldsymbol{k}, k_{\|}$, and $k_{\perp}$ are the wave vector and its components parallel and perpendicular to the magnetic field, $f_{b}$ is the beam ion velocity distribution, and $f_{T e}$ is the longitudinal velocity distribution of the suprathermal electrons. In its derivation, it was assumed that the frequencies and wave vectors of the oscillations lie in the ranges $\omega_{c e} \gg \omega \gg \omega_{c i}$, $\operatorname{Im} \omega \gg \omega_{c i}, k_{\perp} r_{L i} \gg 1 \gg \omega_{c i}, k_{\perp} r_{L e}, \omega \gg k v_{T i}, \omega \gg k_{\|} v_{T e}, v_{T j}$ is the particle thermal velocity of species $j, r_{L j}=v_{T j} / \omega_{c j}$ is the Larmor radius of species $j$. These inequalities imply that the ions may be treated as an unmagnetised plasma whilst the 


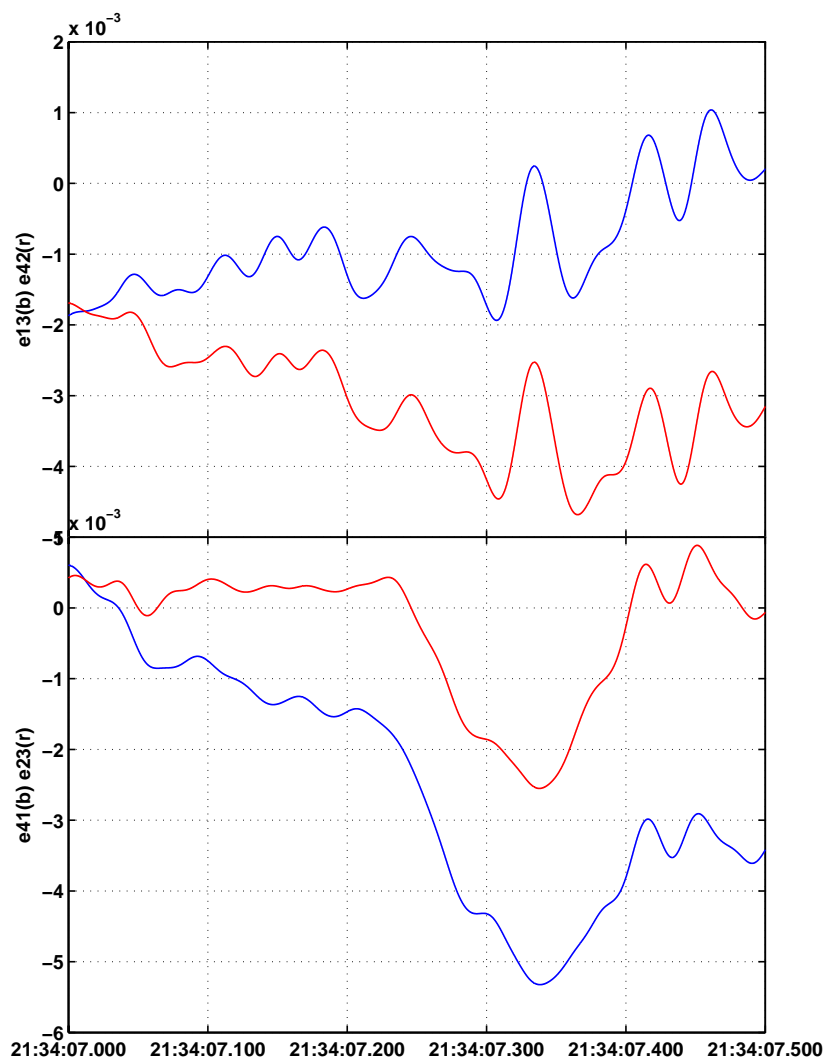

Fig. 13. The electric field waveforms $E_{13}$ and $E_{42}$ (top panel) and $E_{41}$ and $E_{23}$ (lower panel) for the third event.

electrons may be described using the drift approximation. In the limit $\omega_{p e} \gg \omega_{c e}$, which is typical of the bow shock, the oscillations will be electrostatic in nature and occur at the lower-hybrid resonance frequency $\left(k_{\|}=0\right)$ or slightly above $\left(k_{\|} \neq 0\right)$.

The last two terms in Eq. (3) relate to the fact that the waves may be resonant with both the ion and electron populations. The component of the wave vector directed perpendicular to the external magnetic field is able to interact with the ion population, preferentially accelerating the particles in the direction perpendicular to the field direction. The small but finite $k_{\|}$will preferentially accelerate the electron population to higher energies along the field direction.

As was mentioned in Sect. 1, at the terrestrial bow shock lower hybrid mode waves may be generated by the MTSI set up as a result of the incoming solar wind particles interacting with the population of particles that have been reflected at the shock and are thus travelling along the magnetic field lines in the upstream direction. This scenario was used as a basis by Vaisberg et al. (1983) to calculate the average energy of the superthermal tail of the electron distribution as:

$E_{e}=\left(m_{e} / m_{i}\right)^{1 / 5}\left(m_{i} v_{b}^{2}\right)^{2 / 5}\left(m_{i} \Delta v_{b}^{2}\right)^{3 / 5}$

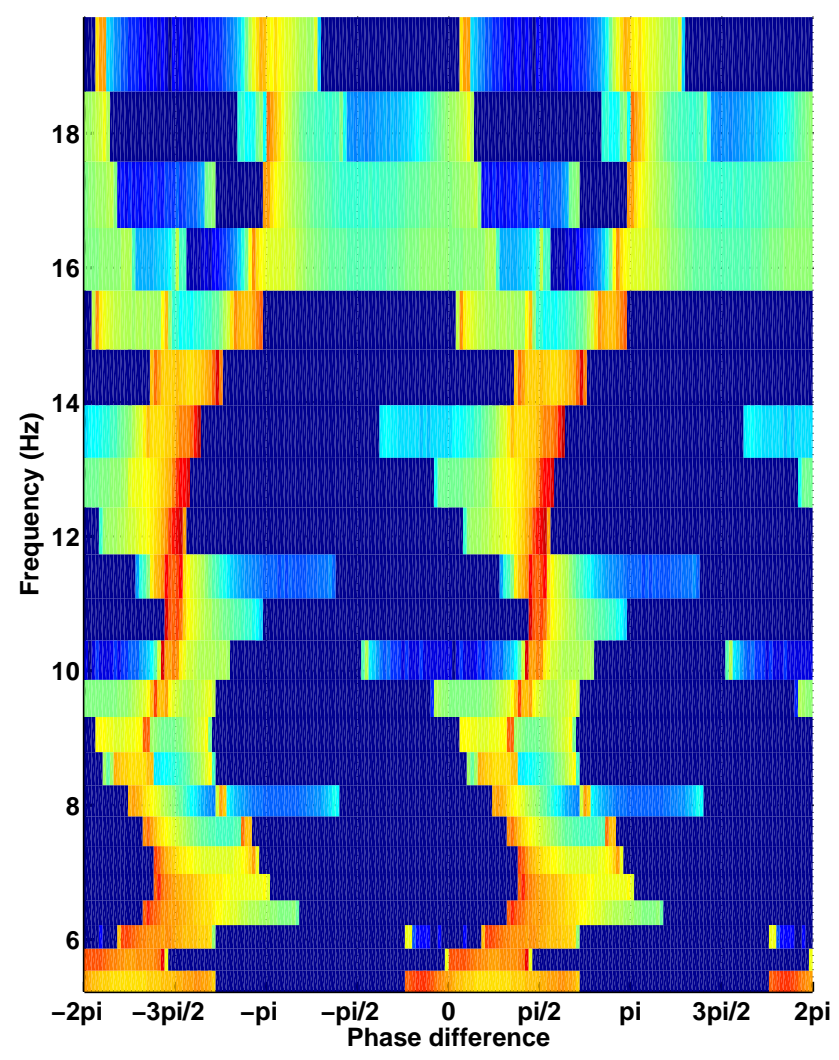

Fig. 14. The $\omega-k$ joint spectrum calculated from the two perpendicular electric field components $E_{13}$ and $E_{41}$ for the third event.

where $m_{e, i}$ are the electron/ion masses, $v_{b}$ is the beam velocity, and $\Delta v_{b}$ is the thermal energy of the beam. Vaisberg et al. (1983) then went on to estimate the distance required in order to accelerate an electron up to this energy and derived Eq. (5).

$$
\begin{aligned}
L_{\|} & =16 \pi^{2} n_{0}^{2} E_{e}^{2}\left(E_{e} / m_{e}\right)^{1 / 2} \\
& \cdot\left[\omega_{L H} H_{0}^{2}\left(\omega_{p e}^{2} / \omega_{c e}^{2}\right) \int\left|E_{k}\right|^{2} d^{3} k /(2 \pi)^{3}\right]^{-1}
\end{aligned}
$$

Using typically observed values values for the beam velocity, the width of its distribution and its density, the average energy of the superthermal tail of the electron distribution calculated using Eq. (4) is $E_{e} \sim 100 \mathrm{eV}$. It should be noted that this values is an average and that a small fraction of particles may be accelerated to very high energies. Again, using the observed magnitudes of the magnetic and electric field, particle density and the average energy of the electrons in the superthermal tail (as calculated above) the size of the spatial region required to accelerate electrons up to these energies may be estimated (from Eq. 5) to be

$$
\begin{aligned}
L_{\|}= & 16 \cdot \pi^{2} \cdot 9.6^{2} \cdot\left(1 \cdot 10^{-7}\right)^{2} \cdot\left(11 \cdot 10^{-7} / 9.1 \cdot 10^{-27}\right)^{1 / 2} \\
\cdot & {\left[63 \cdot\left(1.45 \cdot 10^{-4}\right)^{2} \cdot\left(\left(1.7 \cdot 10^{5}\right)^{2} /\left(2.55 \cdot 10^{3}\right)^{2}\right) .\right.} \\
& \left.\left(1 \cdot 10^{-9}\right)^{2} /(2 \pi)^{3}\right]^{-1} \approx 2500 \mathrm{~km}
\end{aligned}
$$


Thus, to reach energies of $E_{e} \sim 100 \mathrm{eV}$, the acceleration region should have a spatial size of around $2500 \mathrm{~km}$ or greater. In order to provide an efficient acceleration mechanism the spatial region on which lower hybrid waves occur should be of a similar size. However, from our observation, the duration of the lower hybrid wave packets is very short (a few tens of milliseconds) their spatial size is rather small $(\sim 100 \mathrm{~km})$. This size is also much greater than the size of the shock foot region in which the reflected ions are observed.

\section{Conclusions}

This article has presented observations and analysis of waves observed in the frequency range $10-40 \mathrm{~Hz}$ in the foot region of a set of quasiperpendicular shocks. It has been shown that in this region waves are often observed at a frequency just above the local lower hybrid resonance frequency. The analysis shows that most but not all of these wave packets possess linear polarisation which is consistent with the waves propagating in the lower hybrid mode. Some of the wave packets observed in this frequency range possessed circular polarisation and so are whistler mode.

The typical amplitudes of the lower hybrid mode wave packets were determined to be $1-3 \mathrm{mVm}^{-1}$. Using typically observed values for the beam density and energy the average electron energy of the suprathermal population was determined to be of the order of $100 \mathrm{eV}$. In order to provide the necessary acceleration, the lower hybrid waves should occupy a spatial region whose dimension is of the order of $2500 \mathrm{~km}$. However, this is much greater than the region in which the waves are observed, and is also greater than the extent of the observed foot region of a quasiperpendicular shock. As a result it is unlikely that the lower hybrid waves can provide all of the required electron acceleration.

Acknowledgements. The authors wish to acknowledge financial support from PPARC and INTAS. The FGM magnetic field data used in this study were taken from the Cluster Active Archive.

Topical Editor I. A. Daglis thanks M. P. Gough and V. Krasnosel'skikh for their help in evaluating this paper.

\section{References}

Balikhin, M., Walker, S., Treumann, R., Alleyne, H., Krasnoselskikh, V., Gedalin, M., Andre, M., Dunlop, M., and Fazakerley, A.: Ion sound wave packets at the quasiperpendicular shock front, Geophys. Res. Lett., 32, L24 106, doi:10.1029/ 2005GL024660, 2005.

Balikhin, M. A. and Gedalin, M. E.: Comparative analysis of different methods for distinguishing temporal and spatial variations, in: Proc. of START Conf., Aussois, France, vol. ESA WPP 047, pp. 183-187, 1993.

Balikhin, M. A., Dudok de Wit, T., Woolliscroft, L. J. C., Walker, S. N., Alleyne, H., Krasnosel'skikh, V., Mier-Jedrzejowicz, W. A. C., and Baumjohann, W.: Experimental determination of the dispersion of waves observed upstream of a quasiperpendicular shock, Geophys. Res. Lett., 24, 787-790, doi: 10.1029/97GL00671, 1997.

Balogh, A., Dunlop, M. W., Cowley, S. W. H., Southwood, D. J., Thomlinson, J. G., Glassmeier, K. H., Musmann, G., Lühr, H., Buchert, S., Acuña, M. H., Fairfield, D. H., Slavin, J. A., Riedler, W., Schwingenschuh, K., and Kivelson, M. G.: The Cluster magnetic field investigation, Space Sci. Rev., 79, 65-91, 1997.

Gustafsson, G., Bostrøm, R., Holback, B., Holmgren, G., Lundgren, A., Stasiewicz, K., Åéhlen, L., Mozer, F. S., Pankow, D., Harvey, P., Berg, P., Ulrich, R., Pedersen, A., Schmidt, R., Butler, A., Fransen, A. W. C., Klinge, D., Thomsen, M., Falthammar, C.-G., Lindqvist, P.-A., Christenson, S., Holtet, J., Lybekk, B., Sten, T. A., Tanskanen, P., Lappalainen, K., and Wygant, J.: The electric field and wave experiment for the Cluster mission, Space Sci. Rev., 79, 137-156, 1997.

Klimov, S., Savin, S., Aleksevich, Y., Avanesova, G., Balebanov, V., Balikhin, M., Galeev, A., Gribov, B., Nozdrachev, M., Smirnov, V., Sokolov, A., Vaisberg, O., Oberc, P., Krawczyk, Z., Grzedzielski, S., Juchniewicz, J., Nowak, K., Orlowski, D., Parfianovich, B., Wozniak, D., Zbyszynski, Z., Voita, Y., and Triska, P.: Extremely-low-frequency plasma waves in the environment of comet Halley, Nature, 321, 292-293, 1986.

Krasnoselskikh, V. V., Kruchina, E. N., Volokitin, A. S., and Thejappa, G.: Fast electron generation in quasiperpendicular shocks and type II solar radiobursts, Astron. Astrophys., 149, 323-329, 1985.

Krasnosel'skikh, V. V., Balikhin, M. A., Alleyne, H. S., Klimov, S. I., Mier-Jedrzejowicz, W. A. C., Pardaens, A. K., Petrukovich, A., Southwood, D. J., Vinogradova, T., and Woolliscroft, L. J. C.: On the nature of low frequency turbulence in the foot of strong quasi-perpendicular shocks, Adv. Space Res., 11, 15-18, 1991.

Laming, J. M.: Accelerated electrons in cassiopeia A: An explaination for the hard X-ray tail, Astrophys. J., 546, 1149-1158, 2001.

Leroy, M. M., Winske, D., Goodrich, C. C., Wu, C. S., and Papadopoulos, K.: The structure of perpendicular bow shocks, J. Geophys. Res. A, 87, 5081-5094, 1982.

Pinçon, J.-L., Kintner, P. M., Schuck, P. W., and Seyler, C. E.: Observation and analysis of lower hybrid solitary structures as rotating eigenmodes, J. Geophys. Res. A, 102, 17 283-17 296, 1997.

Tjulin, A., Eriksson, A. I., and André, M.: Lower hybrid cavities in the inner magnetosphere, Geophys. Res. Lett., 30, 17-1, doi: 10.1029/2003GL016915, 2003.

Vaisberg, O. L., Galeev, A. A., Zastenker, G. N., Klimov, S. I., Nozdrachev, M. N., Sagdeev, R. Z., Sokolov, A. I., and Shapiro, V. D.: Electron acceleration at the front of strong collisionless shock waves, Zhurnal Eksperimental noi i Teoreticheskoi Fiziki, 85, 1232-1243, 1983.

Walker, S. N., Balikhin, M. A., and Nozdrachev, M. N.: Ramp nonstationarity and the generation of whistler waves upstream of a strong quasiperpendicular shock, Geophys. Res. Lett., 26, 13571360, doi:10.1029/1999GL900210, 1999.

Woolliscroft, L. J. C., Alleyne, H. S. C., Dunford, C. M., Sumner, A., Thompson, J. A., Walker, S. N., Yearby, K. H., Buckley, A., Chapman, S., and Gough, M. P.: The Digital Wave-Processing Experiment on Cluster, Space Sci. Rev., 79, 209-231, 1997. 